\title{
L'Image de la faute dans la légende fondatrice de l'histoire de Normandie à travers les manuscrits de la Grande Chronique de Normandie des XIV et $\mathrm{XV}^{\mathrm{e}}$ siècles
}

Ismérie Triquet

\section{OpenEdition}

\section{Journals}

Édition électronique

URL : http://journals.openedition.org/questes/4227

DOI : $10.4000 /$ questes. 4227

ISSN : 2109-9472

\section{Éditeur}

Les Amis de Questes

\section{Édition imprimée}

Date de publication : 30 octobre 2015

Pagination : 49-64

ISSN : 2102-7188

\section{Référence électronique}

Ismérie Triquet, «L'Image de la faute dans la légende fondatrice de l'histoire de Normandie à travers

les manuscrits de la Grande Chronique de Normandie des XIVe et XVe siècles », Questes [En ligne],

30 | 2015, mis en ligne le 01 novembre 2015, consulté le 30 avril 2019. URL : http://

journals.openedition.org/questes/4227; DOI : 10.4000/questes.4227 


\title{
L'Image de la faute dans la légende fondatrice de l'histoire de Normandie à travers les manuscrits de la Grande Chronique de Normandie des $\mathrm{XIV}^{\mathrm{e}}$ et $\mathrm{XV}^{\mathrm{e}}$ siècles
}

\author{
Ismérie TRIQUET \\ Université Rennes II-Haute Bretagne
}

La littérature médiévale comprend des récits sombres où la justice et la morale ne sont pas au centre des préoccupations et où des personnages négatifs mettent en péril leur propre lignée par leur comportement fautif. La légende de Robert le Diable étant de ces textes, son utilisation comme mythe fondateur du récit d'une importante lignée soulève de nombreuses questions. Ainsi, nous verrons ici dans quelle mesure la faute peut être un élément constitutif d'un mythe fondateur. À travers la légende de Robert le Diable et sa réécriture survenue au $\mathrm{XIV}^{\mathrm{e}}$ siècle, nous verrons comment un mythe fondateur, même négatif, peut s'avérer bénéfique à la mise en valeur d'une lignée seigneuriale. Puis, nous mettrons en parallèle les éléments négatifs de la légende originelle du XII ${ }^{\mathrm{e}}$ siècle et ceux de la version remaniée du XIV ${ }^{\mathrm{e}}$ siècle, version qui introduit du $\mathrm{XIV}^{\mathrm{e}}$ au $\mathrm{XV}^{\mathrm{e}}$ siècle le texte de la Grande Chronique de Normandie. Enfin, nous nous attarderons sur la mise en image de cette légende et sur les partis pris iconographiques retenus pour servir au mieux, non seulement le texte lui-même, mais aussi le récit qu'il introduit, à savoir la Grande Chronique de Normandie, texte à la gloire de la lignée historique normande et donc intimement lié à la légende fondatrice que représente l'histoire de Robert le Diable. 


\section{La légende primitive de Robert le Diable}

$\mathrm{Au}$ XII ${ }^{\mathrm{e}}$ siècle voit le jour un récit anonyme relatant l'histoire légendaire d'un certain Robert le Diable dont le père, Aubert, est duc de Normandie $^{1}$. Son mariage n'étant pas fécond, sa femme, la duchesse Yde, désespérée de prier Dieu en vain pour la venue d'un enfant, se tourne vers le diable qui exauce ses prières ${ }^{2}$. Ainsi naît Robert le Diable, enfant méchant qui violente fréquemment son entourage ${ }^{3}$. Le jeune homme en quête de réponses quant à son comportement questionne sa mère. Celle-ci lui révèle son ascendance diabolique, ce qui pousse Robert à partir chercher la rédemption auprès du pape ${ }^{4}$, puis d'un ermite réputé pour sa sagesse qui est informé par un messager divin de la punition à donner au jeune Robert ${ }^{5}$. En guise de pénitence, il doit se faire passer pour fou, disputer sa nourriture aux animaux et ne plus parler. L'empereur de Rome, rencontré fortuitement, le prend sous son aile au sein de sa cour ${ }^{6}$. Là, Robert s'illustre par des faits chevaleresques anonymes, vêtu d'armes

${ }^{1}$ La version du XII ${ }^{\mathrm{e}}$ siècle est, à ce jour, perdue. Seules nous sont parvenues deux rédactions, l'une dite A (en vers) datant de la seconde moitié du XIII siècle et l'autre dite B (en prose), qui prend place à la fin du XIV siècle. Élisabeth Gaucher, Robert le Diable. Histoire d'une légende, Paris, Honoré Champion, coll. «Essais sur le Moyen Âge », 2003, p. 21.

2 Élisabeth Gaucher, Robert le Diable, Paris, Honoré Champion, coll. «Champion classiques. Moyen Âge », 2006, p. 89-95.

${ }^{3}$ Ibid., p. 97-105.

${ }^{4}$ Ibid., p. 127-131. Sur cette question, voir également les travaux de Régine Colliot, «Un rapport dramatique mère/enfant dans le récit médiéval. La mère dénonciatrice des crimes », dans Les Relations de parenté dans le monde médiéval, dir. Centre universitaire d'études et de recherches médiévales, Aix-en-Provence, CUER MA, coll. «Sénéfiance », 1989, p. 163-176 et Élisabeth Gaucher, «La mère coupable dans la légende de Robert le Diable (XII ${ }^{\mathrm{e}}-\mathrm{XIX}^{\mathrm{e}}$ siècle) », Bien dire et bien aprandre. Revue du Centre de recherches médiévales \& dialectales de Lille III, 16, 1998, p. 133-144.

${ }_{6}^{5}$ Élisabeth Gaucher, Robert le Diable, op. cit., p. 139-145.

${ }^{6}$ Ibid., p. 147-163. En fonction des réécritures, cette folie, par laquelle Robert accomplit sa pénitence, prendra des formes variables, voir Huguette Legros, « La folie de Robert le Diable. Réécritures et spécificités » dans La Légende de Robert le Diable du Moyen Âge au XX $x^{e}$ siècle, dir. Laurence Mathey-Maille et Huguette Legros, Orléans, Paradigme, coll. « Medievalia », 2010, p. 151-174. 
remises par un messager de dieu, alors que l'empereur de Rome est attaqué par les Turcs. L'empereur annonce son souhait de faire de ce chevalier blanc son héritier et de lui donner sa fille en mariage ${ }^{7}$. Mais, une fois découvert, notamment grâce à la fille de l'empereur qui retrouve miraculeusement la parole, Robert refuse cet héritage et se retire auprès de l'ermite où il finit sa vie auréolé de sagesse grâce à sa conversion religieuse $^{8}$. L'histoire se poursuit avec la vie du demi-frère de Robert, Richard, qui mènera une vie exemplaire, notamment en s'illustrant à la croisade avec Charlemagne.

Dans cette version, les actes malveillants de Robert sont rachetés par sa conversion religieuse ${ }^{9}$. Quant à ceux de sa famille, ils sont rachetés par le second mariage d'Aubert, dont naîtra un fils, Richard. En ce qui concerne la première épouse du duc, Yde, nous pouvons noter que, à l'inverse des récits hagiographiques dans lesquels la piété maternelle conditionne la sainteté du héros, l'histoire fait ici de la mater mediatrix un contre-exemple dont le péché va inciter le fils à renier ses origines. Ainsi, dans ce contexte, la mère est aussi coupable que son fils, si ce n'est plus, puisqu'elle est à l'origine du destin de ce dernier. Le récit du XII ${ }^{\mathrm{e}}$ siècle apparaît donc comme un roman d'aventure dans le sens où le lecteur suit les péripéties et les questionnements du héros, mais aussi comme un exemplum, caractérisé par une série de transgressions répétées et ordonnées et où la morale couronne la fin du récit ${ }^{10}$. Enfin, notons que le fantastique, extrêmement présent, contribue à la rédemption du héros en intervenant aux instants où il pourrait douter de la voie choisie.

\footnotetext{
${ }^{7}$ Élisabeth Gaucher, Robert le Diable, op.cit., p. 185-207.

${ }^{8}$ Ibid., p. 209-355.

9 Élisabeth Gaucher-Rémond, «Robert le Diable ou le "criminel repentant". La légende au miroir des récits de conversion », dans La Légende de Robert le Diable, op. cit., p. 27-41.

${ }^{10}$ Pour plus de détails concernant cette version de la légende, voir Élisabeth Gaucher, Robert le Diable. Histoire d'une légende, op. cit., p. 24-69.
} 


\section{La version remaniée du XIV ${ }^{\mathrm{e}}$ siècle}

Comme nous l'avons évoqué précédemment, il existe une seconde version de cette légende, rédigée à la fin du $\operatorname{XIV}^{\mathrm{e}} \operatorname{siècle}^{11}$. Elle se trouve généralement associée à un autre récit, celui racontant l'histoire de la Normandie et connu sous le nom de Grande Chronique de Normandie ${ }^{12}$. Ce texte rédigé vers 1350-1370 est un texte généalogique mettant en valeur l'identité normande des $\mathrm{X}^{\mathrm{e}}-\mathrm{XIII}^{\mathrm{e}}$ siècles ${ }^{13}$. Dans certains manuscrits de la chronique normande, à savoir trente-deux exemplaires sur les quarante-trois connus ${ }^{14}$, une version remaniée de la légende de Robert le Diable a été placée en guise de chapitre introductif ${ }^{15}$.

Dans cette tradition remaniée, la conception de Robert est différente ${ }^{16}$. Aubert rentrant de la chasse souhaitait s'unir à son épouse.

${ }^{11}$ Cette version demeure inédite, le texte n'étant présent que dans certains manuscrits de la Grande chronique de Normandie produite au $\mathrm{XIV}^{\mathrm{e}}$ siècle. Pour plus d'informations sur ce corpus, voir mes travaux de thèse de doctorat: Ismérie Triquet, Les manuscrits enluminés de la Grande chronique de Normandie aux XIV et $X V^{e}$ siècles, Université Rennes II-Haute Bretagne, sous la direction de Bruno Boerner et la co-direction de Denis Hüe, 2014.

12 Le corpus de ces manuscrits a été rassemblé par Gilette Labory, chercheur à l'Institut de Recherche et d'Histoire des Textes (IRHT). Dans un important ensemble de trois articles, elle a dressé des notices relatives à chaque exemplaire : Gilette Labory, «Les manuscrits de la Grande Chronique de Normandie aux XIV ${ }^{\mathrm{e}}$ et $\mathrm{XV}^{\mathrm{e}}$ siècles ", Revue d'histoire des textes, 27, 1997, p. 191-222; Revue d'histoire des textes, 28, 1998, p. 183-233 ; Revue d'histoire des textes, 29; 1999, p. 245-294. C'est Léopold Delisle qui donna son nom à l'œuvre et ce afin de la distinguer des autres chroniques et récits historiographiques très nombreux en Normandie depuis le $\mathrm{X}^{\mathrm{e}}$ siècle.

${ }^{13}$ Le texte relate les faits s'étant déroulés entre 911, date de l'arrivée du Viking Rollon et de la concession de la terre normande faite à ce dernier par le roi Charles le Simple, et 1204, lorsque le roi de France Philippe Auguste reconquiert la Normandie et la rattache à sa couronne.

${ }^{14}$ Plus six exemplaires perdus dont la trace demeure uniquement dans les catalogues anciens.

${ }^{15}$ Un second chapitre introductif accompagne la légende de Robert le Diable : il s'agit du récit des aventures du pirate viking Hasting qui ravagea les terres françaises avant l'arrivée de Rollon.

${ }^{16}$ Sur cette version remaniée, voir Gillette Labory, « Réflexion sur le remaniement de la Grande Chronique de Normandie à la fin du XV siècle ", dans Guerre, pouvoir et noblesse au Moyen Âge. Mélanges en l'honneur de Philippe Contamine, 
Celle-ci se refusa à lui et ce dernier la contraignit à l'acte sexuel. Ainsi, dans cette situation, les deux époux font preuve d'hybris et c'est cette faute qui est à l'origine de l'histoire de Robert le Diable. C'est donc dans la violence que Robert fut conçu, mais ce n'est pas ce qui conditionna son caractère. Ce dernier se développa dès sa petite enfance et ne fit que croître durant la première moitié de la vie du jeune homme. Outre la faute de ses parents, l'histoire recèle de nombreux autres méfaits. En effet, dans son enfance, Robert creva les yeux de ses camarades d'école, puis poignarda son professeur qui l'avait battu. Voyant les méfaits de son fils s'aggraver, le duc décida, sur les conseils de son épouse, de le faire chevalier, pensant que la chevalerie et toute la noblesse qui accompagne ce statut lui permettraient de revenir dans le droit chemin. Cependant, dès les préparatifs de la cérémonie tout se déroula au plus mal. Au lieu de veiller toute la nuit en prière, Robert viola et tua plusieurs religieuses de l'abbaye d'Arques avant d'aller se coucher couvert de sang. Au matin, il préféra aller se restaurer au lieu de se rendre à la messe. Lors de la cérémonie, le jeune homme se saisit de l'épée que son père venait de lui remettre et tenta de le frapper, ajoutant la tentative de parricide à ses crimes.

Dans cette version, il n'y a pas d'aveu de sa mère Yde. C'est donc de sa propre initiative que Robert décide de quitter la Normandie, assassinant en chemin le fils du vicomte de Coutances qui réclamera vengeance et traquera Robert. Devant ces événements, Aubert, le père de Robert, annonça qu'il pardonnerait à quiconque tuerait son fils. C'est donc blessé et traqué que Robert quitta la terre de son père. Dans sa fuite, il jeta ses armes et rencontra un ermite à qui il demanda le gîte. Ce dernier le soigna et lui conseilla de revenir vers Dieu en faisant preuve de 
pénitence et de contrition et il lui donna sa bénédiction et l'absolution pour ses péchés. Le jeune homme fixa lui-même les termes de sa pénitence. Une fois guéri, Robert se mit en chemin pour aller rencontrer le pape afin que sa pénitence soit complétée et fixée à une durée de sept ans. Enfin, Robert trouva refuge à la cour de l'empereur de Rome où il exécuta sa pénitence et mourut pieusement. La duchesse Yde, désespérée de l'attitude de son fils, ne décoléra pas de toute sa vie et en mourut.

La seconde partie du texte met l'accent sur le second mariage du duc Aubert avec Berthe. De cette union naîtra Richard, ainsi qu'une fille qui n'est pas nommée. Richard s'avérera être un valeureux chevalier qui s'illustrera aux côtés de Charlemagne lors de la croisade. Aucun des descendants mâles ne donna d'enfant à la lignée. Seule la fille issue de la seconde union d'Aubert donnera un fils au duché. Cet enfant sera un garçon, prénommé Ernais. Il tentera d'usurper la couronne de France et sera assassiné comme un traître par Guillaume au Court Nez. Avec la mort de ce dernier, le duché de Neustrie, se trouvant sans héritier, sera rattaché à la couronne de France jusqu'au règne de Charles le Simple et l'arrivée de Rollon.

Dans cette version, le récit se focalise sur l'aventure individuelle des personnages, sur leur vie intérieure, au détriment des faits de chevalerie, s'éloignant ainsi du genre des biographies chevaleresques. Pour accréditer son discours, l'auteur de la Grande Chronique de Normandie n'hésite pas à rejeter les «vrayes croniques» qui «racontent que Rou fut le premier duc de Normandie ${ }^{17}$. Lui-même s'autorise à qualifier son récit de source en insistant sur le fait qu' «aucune autre escriptures $»^{18}$. Quoi qu'il en soit, l'auteur fait largement appel à la

17 Grande chronique de Normandie, Rouen, bibliothèque municipale, Incunable mm23, p. 13

${ }^{18}$ Ibid., p. 13 ; Élisabeth Gaucher, Robert le Diable. Histoire d'une légende, op. cit., p. 101. 
fiction. Il est indéniable qu'il connaissait la légende de Robert le Diable dans sa forme originelle, car c'est sur elle qu'il fonde son récit, mais il y apporte des modifications, essentiellement d'ordre théogonique. Les éléments généalogiques trop fantaisistes sont évacués, notamment parce qu'ils risquaient d'évoquer l'influence d'une fiction du XII ${ }^{\mathrm{e}}$ siècle, le Roman du Comte de Poitiers ${ }^{19}$. Ainsi, dans la légende de Robert le Diable, Yde est rattachée à la lignée de Bourgogne ${ }^{20}$. Les éléments conservés sont destinés à placer les personnages dans un contexte réaliste $^{21}$ : Aubert devient duc de Neustrie, installé avec sa cour dans la ville de Rouen. Nous pouvons donc noter la volonté très forte d'historiciser la légende afin de lui donner plus de crédibilité. Ce point est encore accentué par l'utilisation de noms fréquemment rencontrés dans les chansons de geste et les divers textes épiques en vogue au XIV siècle, où les généalogies mythiques fonctionnent comme des références obligées, répondant ainsi aux exigences d'un public friand du topos de l'imaginaire fabuleux de la noblesse à la fin du Moyen Âge.

Dans ce récit remanié, les deux parents sont coupables d'hybris : il s'agit d'une faute fondamentale qui peut, dans les sociétés chrétiennes, être mise en parallèle avec le pêché. L'hybris est ici caractérisée par la colère de la duchesse et la sexualité ardente du duc. Dans leurs ébats, le nom du diable n'est pas prononcé, mais les époux ne souhaitent pas que Dieu prenne part à leur union ${ }^{22}$. Ainsi, le héros doit son nom, non pas à une ascendance diabolique comme dans la version originelle mais à une hérédité humaine, et plus tard à ses actes mauvais. La faute primitive des parents est poursuivie par leur descendance. Le chroniqueur surenchérit

\footnotetext{
${ }^{19}$ Ibid., p. 102.

${ }^{20}$ Elle est ici présentée comme la sœur du duc de Bourgogne.

${ }^{21}$ Ibid., p. 102.

${ }^{22}$ Ibid., p. 103.
} 
sur les «mâles enfances ${ }^{23}$ » de Robert et sur les fautes qu'il a commises durant sa jeunesse. Nous pouvons notamment relever les sévices qu'il fit subir aux enfants de sa classe, en leur crevant les yeux ou encore lorsqu'il poignarda son professeur en représailles d'une correction qu'il avait reçue. En grandissant, ses actes deviennent de plus en plus sacrilèges. Alors que son père prit la décision de l'adouber, le jeune homme revisita le rituel de manière particulièrement violente en le marquant d'un sceau ensanglanté et blasphématoire. C'est cet acte, et ceux qui suivront, qui initient la rupture entre le père et le fils. Ainsi la Grande Chronique de Normandie est centrée sur le conflit familial et $\operatorname{social}^{24}$ : Robert attaqua tous les chevaliers du duché qu'il rencontra, amenant ainsi la vengeance sur sa personne, si bien que son père décida de pardonner à quiconque mettrait à mort ce fils gênant ${ }^{25}$. Cet acte est révélateur de la violence qui anime les personnages et, par là même, accroît le caractère négatif du récit. En supprimant la notion de déterminisme surnaturel mais non l'inquiétante renommée du héros, le chroniqueur donne à la légende une plus grande vraisemblance : celle-ci garantit l'authenticité historique du récit dynastique tout en favorisant le didactisme moral puisque l'être diabolique, désormais plus proche des hommes, acquiert par sa conversion au bien une exemplarité accessible qui ne décourage pas l'imitation.

Le remaniement du XIV ${ }^{\mathrm{e}}$ siècle a été pensé de manière à donner au texte une réalité historique qui puisse servir la nostalgie identitaire de la

${ }^{23}$ Ibid., p. 103 et Élisabeth Gaucher, «Enfances diaboliques. Robert le Diable », dans Enfances romanesques, dir. Équipe de recherche sur la littérature d'imagination du Moyen Âge, Poitiers, ERLIMA, 1996, p. 17-26.

24 Jacques Berlioz, «Dramma di famiglia e ideale cavalleresco. La leggenda di Roberto il Diavolo nelle 'Chroniques de Normandie' (XIV secolo) », dans $L a$ Paura dei padri nella società antica e medievale, dir. Ezio Pellizer et Nevio Zorzetti, Rome/Bari, Laterza, coll. «Biblioteca di cultura moderna Laterza », 1983, p. 155-169.

${ }^{25}$ Élisabeth Gaucher, Robert le Diable. Histoire d'une légende, op. cit., p. 103. 
dynastie ducale normande développée par la Grande Chronique de Normandie, même si cette réalité demeure toute relative. Ce type de récit relève d'un courant qui remonte à la littérature généalogique des $\mathrm{XI}^{\mathrm{e}}-$ $\mathrm{XII}^{\mathrm{e}}$ siècles, où le héros fondateur est inventé de toute pièce ${ }^{26}$. Les emprunts à la fiction légendaire contribuent à auréoler le lignage d'un passé mythique. Dans ces récits, une lignée, même fondée sur la violence, se poursuivait de père en fils sans rupture, mais dans la version du $\mathrm{XIV}^{\mathrm{e}}$ siècle le projet échoue car la lignée s'éteint.

Nous pouvons dès lors nous poser la question de l'utilité d'une introduction aussi négative pour servir la dynastie ducale normande. Pour éclaircir ce point, il faut considérer les manuscrits de la Grande Chronique de Normandie dans leur ensemble. En effet, le texte relatif à Robert le Diable doit être mis en rapport avec le début du texte de la chronique à proprement parler. Alors que, dans la tradition originelle, les fautes de Robert et de ses parents sont rachetées par la figure de Richard, dans la version remaniée cela se passe différemment. Même si Richard y apparaît comme un personnage valeureux, il n'apporte aucune descendance à la lignée. Seule sa sœur donne un héritier au duché, mais il tentera d'usurper la couronne de France. Ainsi la légende remaniée est définitivement négative, les actes dommageables des personnages seront rachetés par l'arrivée du Viking Rollon en Neustrie. Il est important de noter que la figure de Rollon est déjà mise en parallèle avec un personnage négatif dans les premiers temps de la chronique : le pirate Hasting qui dévasta les contrées lors des premières invasions vikings. Certaines figures font double emploi et il devient de ce fait difficile de saisir l'importance de certains personnages par rapport aux autres. Nous

${ }^{26}$ Georges Duby, «Remarques sur la littérature généalogique en France aux XI et XII ${ }^{\mathrm{e}}$ siècles », Comptes rendus des séances de l'Académie des Inscriptions et Belles-Lettres, 2, 1967, p. 335-345. 
pourrions, de prime abord, penser que ce sont les actes de Robert qui sont développés pour mettre en valeur la légitimité de Rollon sur la terre de Neustrie. Or, c'est Ernais, le petit-fils du duc Aubert, qui apparaît comme le contrepoids négatif du premier duc de Normandie, répondant ainsi à la justification du bon Viking fondateur d'une lignée légitime. Dans tous les cas, la première lignée ayant failli, il convenait qu'elle fût remplacée par une autre plus méritante. D'un point de vue plus historique, le jeune chef viking acquiert cette légitimité par sa conversion au catholicisme et par les termes conclus durant le traité de Saint-Clair-sur-Epte en $911^{27}$. En effet, à l'occasion de ce traité, le roi de France Charles le Simple, ne pouvant repousser les Vikings, fut contraint de leur concéder un territoire au sein du royaume de France : la Neustrie, qui deviendra la Normandie. Rollon s'est ainsi engagé à protéger le royaume de toute future invasion scandinave, à se convertir, lui et ses hommes, au catholicisme et à épouser la fille du roi, Gisèle ${ }^{28}$.

Finalement la version remaniée qui introduit la Grande Chronique de Normandie apparaît être le récit d'un échec familial, le faux départ d'une grande dynastie ${ }^{29}$. C'est l'association au texte de la Grande Chronique de Normandie qui donne tout son sens à ce chapitre introductif. Ce remaniement textuel était cependant nécessaire pour remplacer les écrits de Dudon de Saint-Quentin ${ }^{30}$ afin qu'ils soient plus crédibles et plus en accord avec la littérature de la fin du Moyen Âge.

27 François Neveux, L'Aventure des Normands, VIII $-\mathrm{XIII}{ }^{\mathrm{e}}$ siècle, Paris, Perrin, coll. « Tempus », 2006, p. 67-72.

${ }^{28}$ Il existe peu de preuves que cette union ait vraiment eu lieu. Ce dont nous sommes certains, c'est que Rollon aura pour frilla, épouse à la mode danoise, une dénommée Poppa.

${ }^{29}$ Élisabeth Gaucher, Robert le Diable. Histoire d'une légende, op. cit., p. 105.

${ }^{30}$ Dudon, De moribus et actis primorum Normanniae ducum, Ed. Jules Lair, Caen Le Blanc-Gardel, 1865. 


\section{La mise en image de la version remaniée au $\mathrm{Xv}^{\mathrm{e}}$ siècle}

Seuls deux manuscrits qui illustrent le récit originel du XII ${ }^{\mathrm{e}}$ siècle nous sont parvenus. Dans une miniature tirée du manuscrit Paris, BnF, fr. 25 516, provenant du Nord de la France, daté des XIII ${ }^{\mathrm{e}}-\mathrm{XIV}^{\mathrm{e}}$ siècles $^{31}$ et comptant onze miniatures, est représenté l'ange qui remet l'armure blanche à Robert sous le regard de la fille de l'empereur. Dans une illustration du manuscrit Paris, BnF, fr. 24 405, qui en contient deux et qui est également originaire du Nord de la France et daté des XIV $\mathrm{XV}^{\mathrm{e}}$ siècles $^{32}$, ce sont les méfaits de jeunesse de Robert qui sont illustrés. Mais ces cycles iconographiques sont centrés sur les aspects fantastiques du récit et n'ont donc pas influencé la mise en image de la fin du Moyen Âge.

Concernant la version remaniée, les partis-pris iconographiques sont différents. Sur les trente-deux manuscrits de la Grande Chronique de Normandie renfermant cette version du récit de Robert le Diable, seuls quatre présentent une illustration relative à cette introduction. Toutes ont été réalisées au $\mathrm{XV}^{\mathrm{e}}$ siècle. La première miniature, probablement une des plus importantes pour notre étude, appartient au manuscrit de la British Library de Londres conservé sous la cote Royal $15 \mathrm{E} \mathrm{VI}^{33}$. Cet exemplaire flamand, daté des années 1445, présente une illustration singulière de la légende de Robert le Diable qui peut être interprétée de plusieurs façons. La miniature prend la forme d'une fresque narrative où l'histoire se développe de gauche à droite. À gauche, deux cavaliers se dirigent vers le centre de la composition qui est occupée par une structure architecturale ouverte au sein de laquelle prend place un couple de personnes alitées. Puis, vers la droite, un groupe de cavaliers sort de

\footnotetext{
${ }^{31}$ Fig. 1.

32 Fig. 2.

${ }^{33}$ Fig. 3.
} 
l'édifice pour se diriger vers un second bâtiment où un homme richement vêtu semble les attendre. Quant à la scène centrale, celle qui se déroule dans le premier bâtiment, nous pouvons supposer que cette partie de l'image met en scène le viol de la duchesse Yde par le duc Aubert. Il s'agit là d'une représentation traditionnelle du lit conjugal et de la conception d'un enfant, mais ici nous ne trouvons aucune présence de Dieu comme traditionnellement dans les scènes de procréation. De plus, le duc est encore habillé alors que son épouse est nue, coiffée de blanc et couchée, symbole de la domination de l'homme sur son épouse ${ }^{34}$. Toutefois, il existe une autre explication à cette image : peut-être s'agit-il de l'illustration du second mariage d'Aubert. En effet, la partie droite de l'image semble davantage relative à la vie de Richard se rendant auprès de Charlemagne, le personnage placé à l'extrême droite. Néanmoins, il est possible de penser que cette partie de l'image relate le départ de Robert et sa rencontre avec l'empereur de Rome, en raison de la richesse vestimentaire du personnage de droite. La scène est difficile à identifier. Toutefois, il est à noter que la vie conjugale du duc Aubert se trouve au centre de la composition, et qu'elle traite de son premier mariage désastreux ou du second plus harmonieux, signe que ce point de l'histoire est au centre de la légende fautive de Robert le Diable. Ainsi, c'est soit la faute originelle qui est représentée, soit son rachat lors des secondes noces ducales. Quoi qu'il en soit, la notion de faute reste présente dans cette image.

Dans une autre image de notre corpus, placée au folio $1 \mathrm{du}$ manuscrit Paris, BnF, fr. $2623^{35}$, l'artiste nous présente une enluminure à plusieurs registres relatant la vie de Robert le Diable. Ce manuscrit daté

\footnotetext{
${ }^{34}$ Florence Colin-Goguel, L'Image de l'amour charnel au Moyen Âge, Paris, Seuil, 2008, p. 140.

${ }^{35}$ Fig. 4. 
des années 1460 est attribué au Maître de l'Échevinage, chef de file de l'enluminure rouennaise des $\mathrm{XIV}^{\mathrm{e}}$ et $\mathrm{XV}^{\mathrm{e}}$ siècles. En haut à gauche de la composition, l'artiste a représenté la duchesse alors qu'elle se refuse à son époux. Nous pouvons voir clairement l'insistance du duc qui étreint sa femme et le refus de celle-ci qui tente de le repousser. Juste à côté de cette scène est traité le passage relatif à la naissance de Robert le Diable : alors que la duchesse est alitée, la nourrice porte l'enfant dans ses bras. En bas à gauche, nous assistons au meurtre du professeur qui est égorgé par le jeune Robert en présence des autres élèves. Enfin, le grand registre de droite représente l'adoubement de Robert par son père. La cérémonie touche à sa fin et le jeune chevalier brandit déjà son épée pour tuer son père mais il est retenu par des nobles qui assistent à l'adoubement. Au centre de l'image, deux personnages anonymes indiquent au public le sens de lecture de l'image. Cette miniature regroupe tous les méfaits de la vie de Robert le Diable jusqu'à son départ du duché, la faute de ses parents étant clairement identifiable comme préambule de la légende.

D'autres manuscrits du corpus présentent des cycles d'images moins complets. Ainsi est-il intéressant de voir les choix iconographiques qui ont été faits lors de la réalisation de ces ouvrages. Dans le manuscrit Paris, BnF, fr. $5388^{36}$, deux thèmes de la légende ont été regroupés dans une seule et même image. Cet exemplaire parisien, daté du premier quart du Xv ${ }^{\mathrm{e}}$ siècle, a été exécuté dans les ateliers du Maître de l'Apocalypse de Jean de Berry. Dans le registre de gauche nous retrouvons la scène du meurtre du professeur. Les personnages sont rassemblés à l'extrême gauche de la composition, laissant un espace vide où a été représenté un livre, rappel de la fonction de l'homme en train de se faire égorger. Les deux scènes de l'image sont séparées par une sorte de muret bas, résidu d'un édifice qui sert à signifier que le meurtre se déroule dans un espace

\footnotetext{
${ }^{36}$ Fig. 5.
} 
fermé. Dans la partie droite de l'image, nous retrouvons l'adoubement de Robert. Le jeune homme ne se fait pas encore menaçant mais les témoins placés derrière lui semblent émettre des doutes quant à la suite paisible de la cérémonie : leurs mains sont levées et leurs regards détournés, certains semblent converser. Dans cet ouvrage, l'accent est mis sur les actes négatifs de Robert vis à vis des représentants de l'autorité.

Enfin nous pouvons noter, au sein des manuscrits de la Grande Chronique de Normandie, un témoin présentant plusieurs illustrations indépendantes de la vie de Robert le Diable. Il s'agit du manuscrit Rouen, bibliothèque municipale, Y 26, qui a été réalisé entre 1410 et 1420 dans les milieux artistiques parisiens. Dans cet ouvrage, trois illustrations sont rattachées à l'histoire de la vie de Robert. La première, au folio $6 \mathrm{r}^{37}$, représente le duc Aubert à sa cour. La scène n'apporte que peu d'éléments quant à la mise en lumière du texte. Cette image peut être perçue comme une ouverture du récit qui n'est pas la plus importante du cycle en raison de son absence d'action significative. Pour la deuxième, l'artiste a, lui aussi, choisi de représenter la scène de l'adoubement de Robert $^{38}$. On y retrouve les mêmes caractéristiques que celles vues dans le manuscrit Paris, BnF, fr. $5388^{39}$, c'est-à-dire que nous sommes au début de la cérémonie et que seules la mise en retrait et la gestuelle des témoins semblent suggérer une menace. La faute avec la tentative de parricide est imminente mais n'a pas encore eu lieu.

La troisième image traite d'événements que nous n'avons pas encore rencontrés : il s'agit des suites du meurtre du fils du vicomte de Coutances par Robert ${ }^{40}$. Le jeune Robert est retrouvé par le père de l'enfant assassiné et violemment frappé. Il s'agit de la seule scène

\footnotetext{
${ }^{37}$ Fig. 6.

${ }^{38}$ Fig. 7.

${ }^{39}$ Fig. 5.

${ }^{40}$ Fig. 8.
} 
illustrant clairement la vie de Robert lors de son départ du château familial. Robert est plutôt dans une position défensive qu'offensive : une de ses victimes est déjà à terre et l'entourage du vicomte de Coutances attaque Robert à trois contre un. Le jeune homme brandit son arme pour se défendre mais il reçoit un coup d'épée dans le flanc, blessure qui le contraindra à fuir. La scène est très violente et semble sonner le glas de la vie diabolique de Robert puisqu'il s'agit là de son dernier méfait. Cette image clôt le cycle iconographique de ce manuscrit qui semble quelque peu décousu au regard de ceux vus précédemment. Finalement, aucune illustration du repentir de Robert n'a été retenue par les enlumineurs, le personnage reste profondément négatif d'autant plus que son pendant positif, Richard, n'est que peu développé.

La faute dans la légende de Robert le Diable occupe une place d'importance, tant dans le texte originel que dans sa version remaniée. Toutefois, la tradition peut être perçue de manière totalement différente d'une version à l'autre. Alors qu' au XII ${ }^{\mathrm{e}}$ siècle, seule sa mère est en cause, le récit du XIV siècle fait des deux époux les coupables du destin de leur fils. De plus, alors qu'au XII' siècle il n'existe qu'une seule faute qui aura des conséquences durant la vie de Robert, la version remaniée nous présente plusieurs éléments fautifs tout au long de l'histoire. En acquérant une dimension historique, la fonction du texte gagne en importance. En partant d'un récit isolé, teinté d'un caractère dynastique, l'auteur du remaniement donne une réelle utilité au texte qui manque cependant de cohésion. En effet, la multiplication des modèles négatifs (Robert et Hasting) censée répondre au modèle positif de Rollon dessert parfois la volonté de l'auteur de glorifier la dynastie normande. De plus, cela risque de perdre le lecteur s'il ne s'attarde pas sur la personnalité et la fonction de chacune des figures. L'illustration, pour sa part, ne met pas en 
parallèle Rollon et Robert, si ce n'est en relatant leur histoire l'une à la suite de l'autre. Ainsi, dans les manuscrits dépourvus de ce chapitre introductif, Rollon bénéficie déjà d'un contre-modèle négatif en la figure de Hasting. De ce fait, nous pouvons nous poser la question de la réelle utilité du récit légendaire et de sa mise en image. De plus, sans pour autant être occultée, la faute primaire de la légende de Robert le Diable, à savoir sa conception, est généralement remisée au profit de l'illustration des méfaits de sa jeunesse et ce toujours dans l'idée de répondre aux actes valeureux de Rollon sans pour autant que cela s'avère nécessaire. 


\section{Cahier d'illustrations}

Ismérie TrIQuET, «L'image de la faute dans la légende fondatrice de l'histoire de Normandie à travers les manuscrits de la

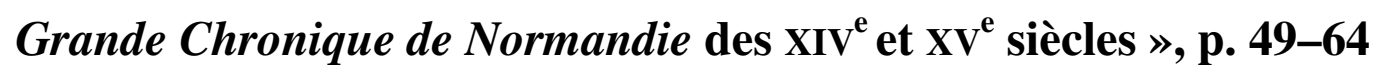

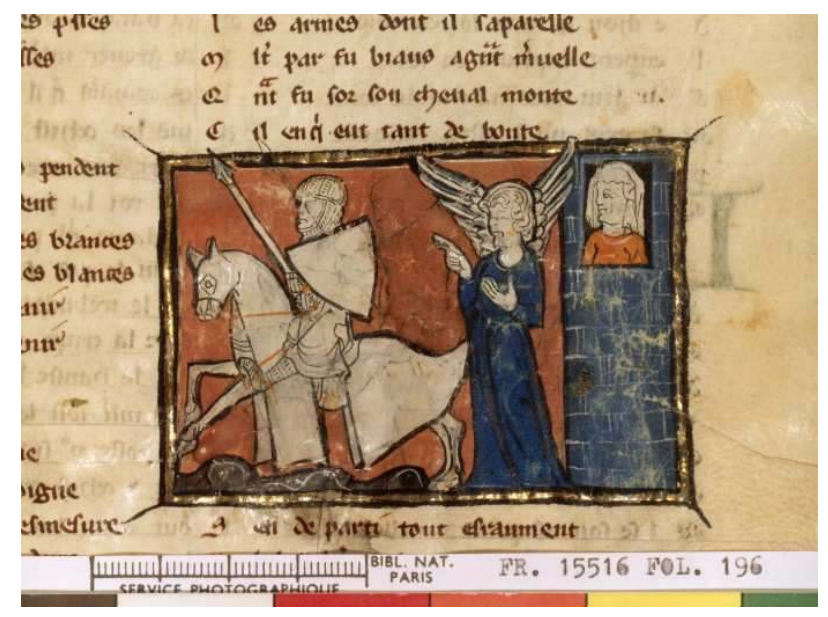

Figure 1 - Robert le Diable, Paris, BnF ms fr. 25 516, XIII-XIV ${ }^{\mathrm{e}}$. L'ange donne une armure blanche à Robert le Diable sous les yeux de la princesse

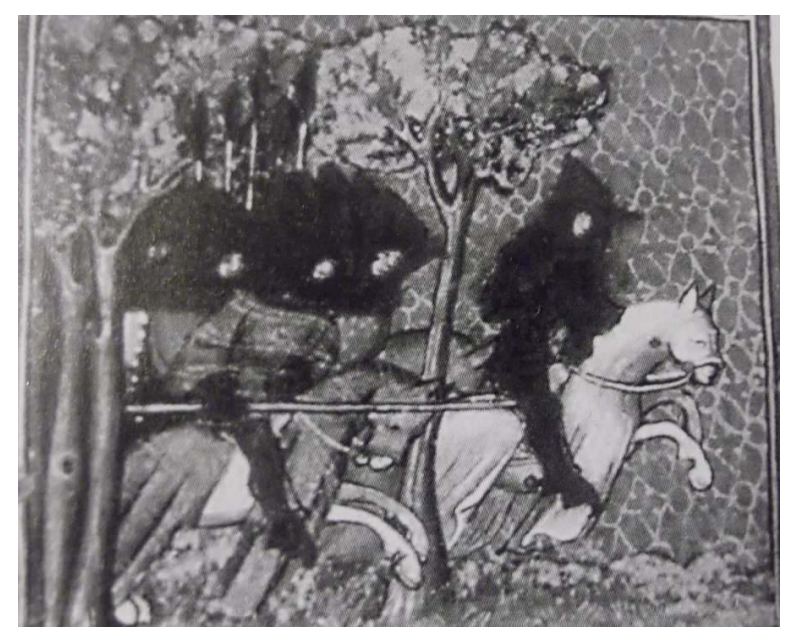

Figure 2 - Robert le Diable, Paris, BnF ms fr. 24 405, $\mathrm{XIV}^{\mathrm{e}}-\mathrm{XV}^{\mathrm{e}}$. Robert le Diable chevauchant avec ses compagnons criminels. 


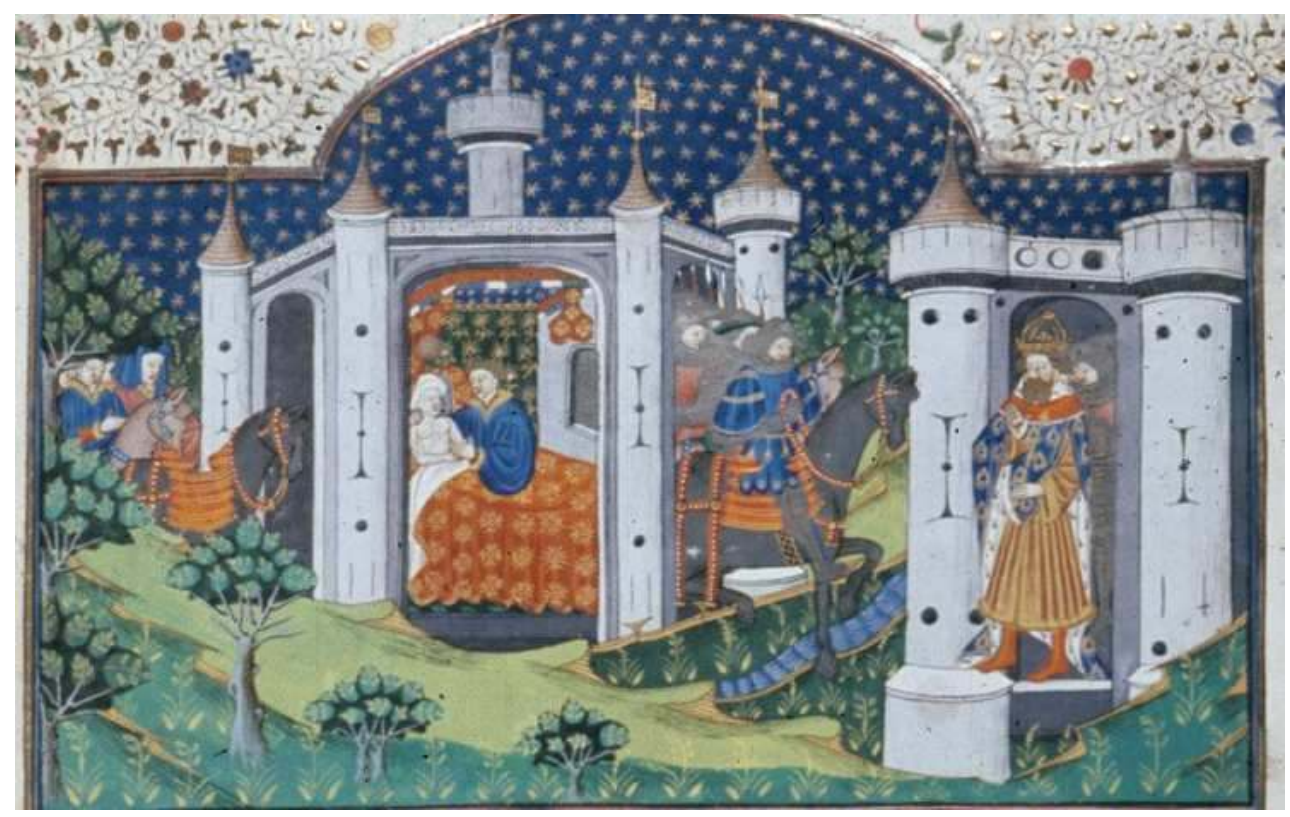

Figure 3 - The Shrewsbury Book, Grande Chronique de Normandie, Londres, British Library ms Royal 15 E VI, fol. 363 r, vers 1445, Normandie, Maître de John Talbot. La légende de Robert le Diable

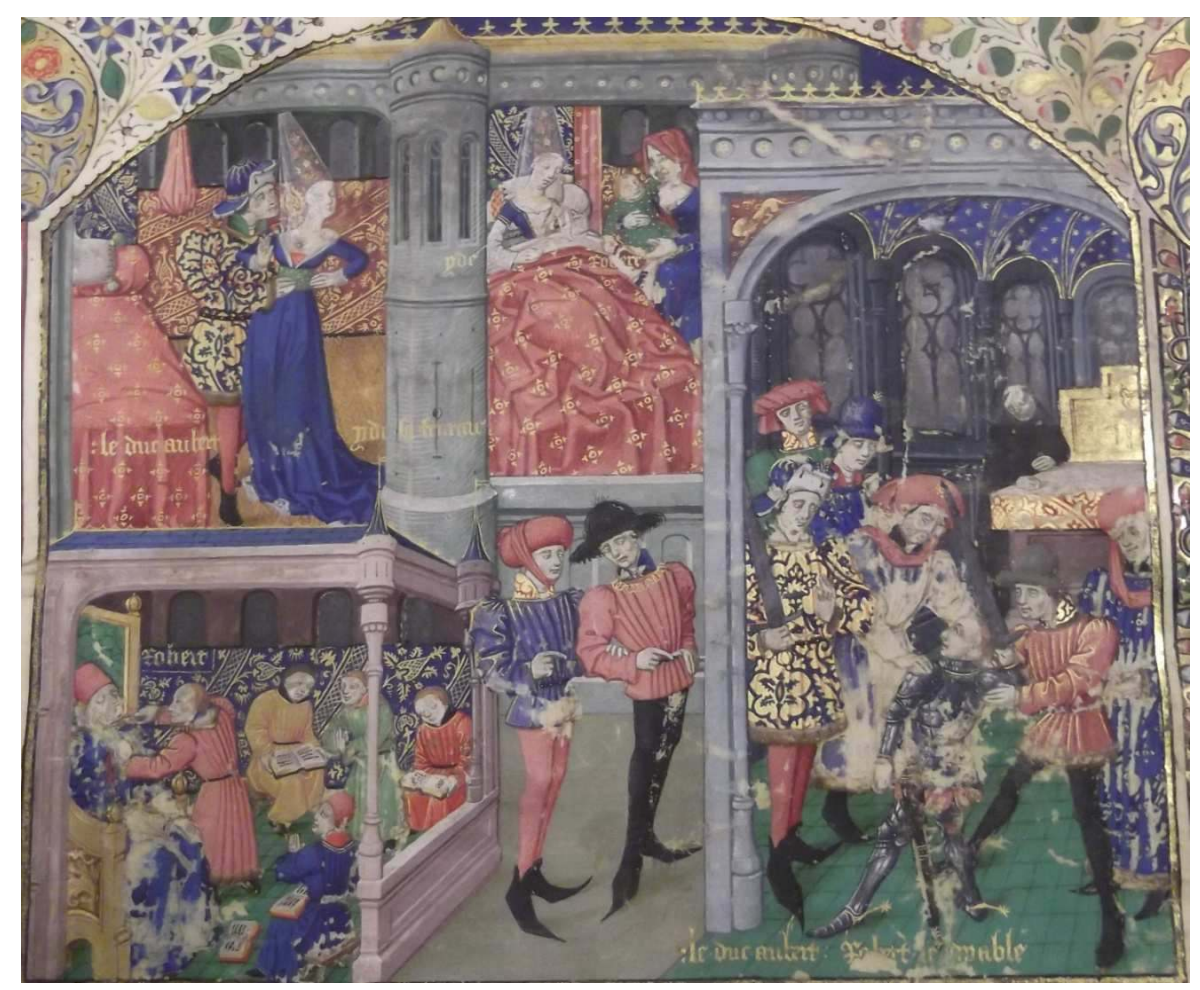

Figure 4 - Maître de l'Echevinage de Rouen, Grande Chronique de Normandie, Paris, BnF ms fr. 2 623, fol. 1r, vers 1460, Rouen, Légende de Robert le Diable. 


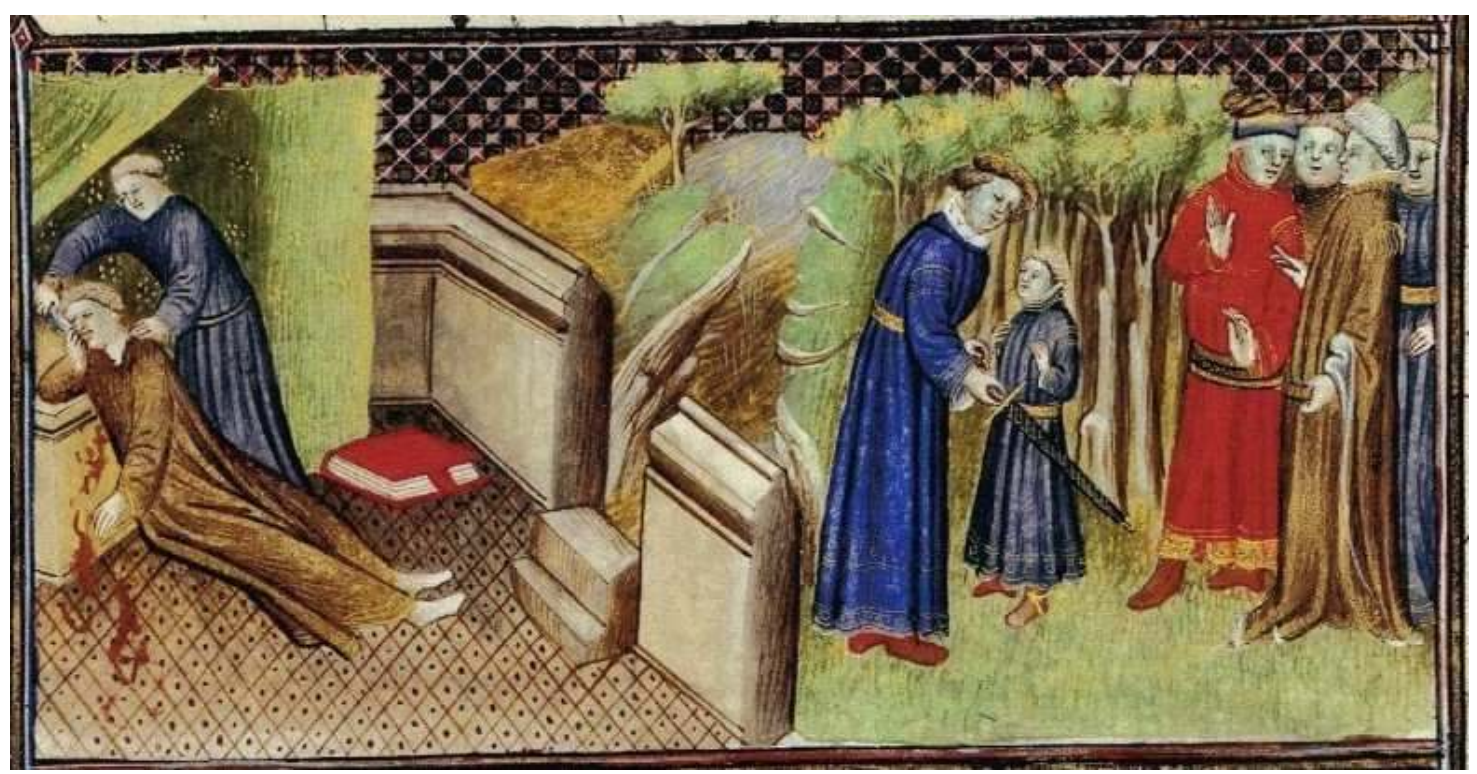

Figure 5 - Maître de l'Apocalypse de Jean de Berry, Grande Chronique de Normandie, Paris, BnF ms fr. 5 388, fol. 10r, premier quart $\mathrm{du} \mathrm{XV}^{\mathrm{e}}$ siècle, Paris, Légende de Robert le Diable, le meurtre du professeur et l'adoubement de Robert.

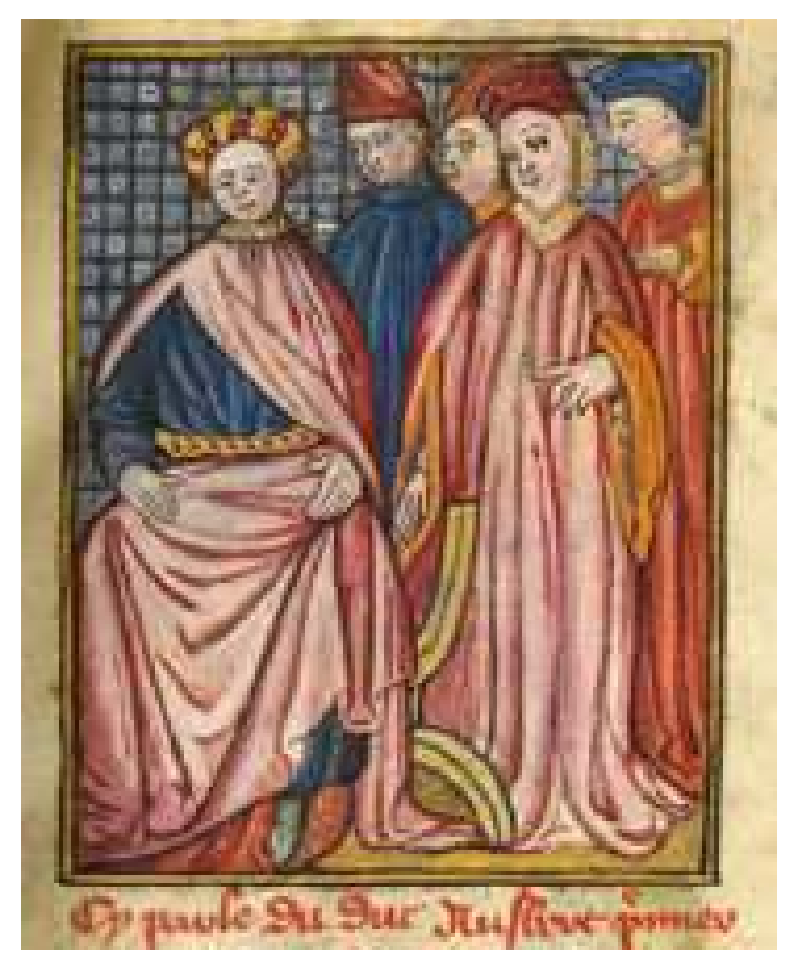

Figure 6 - Grande Chronique de Normandie, Rouen, ms Y26, fol. 6r, 1410-1420, Paris. Le duc Aubert et sa cour. 


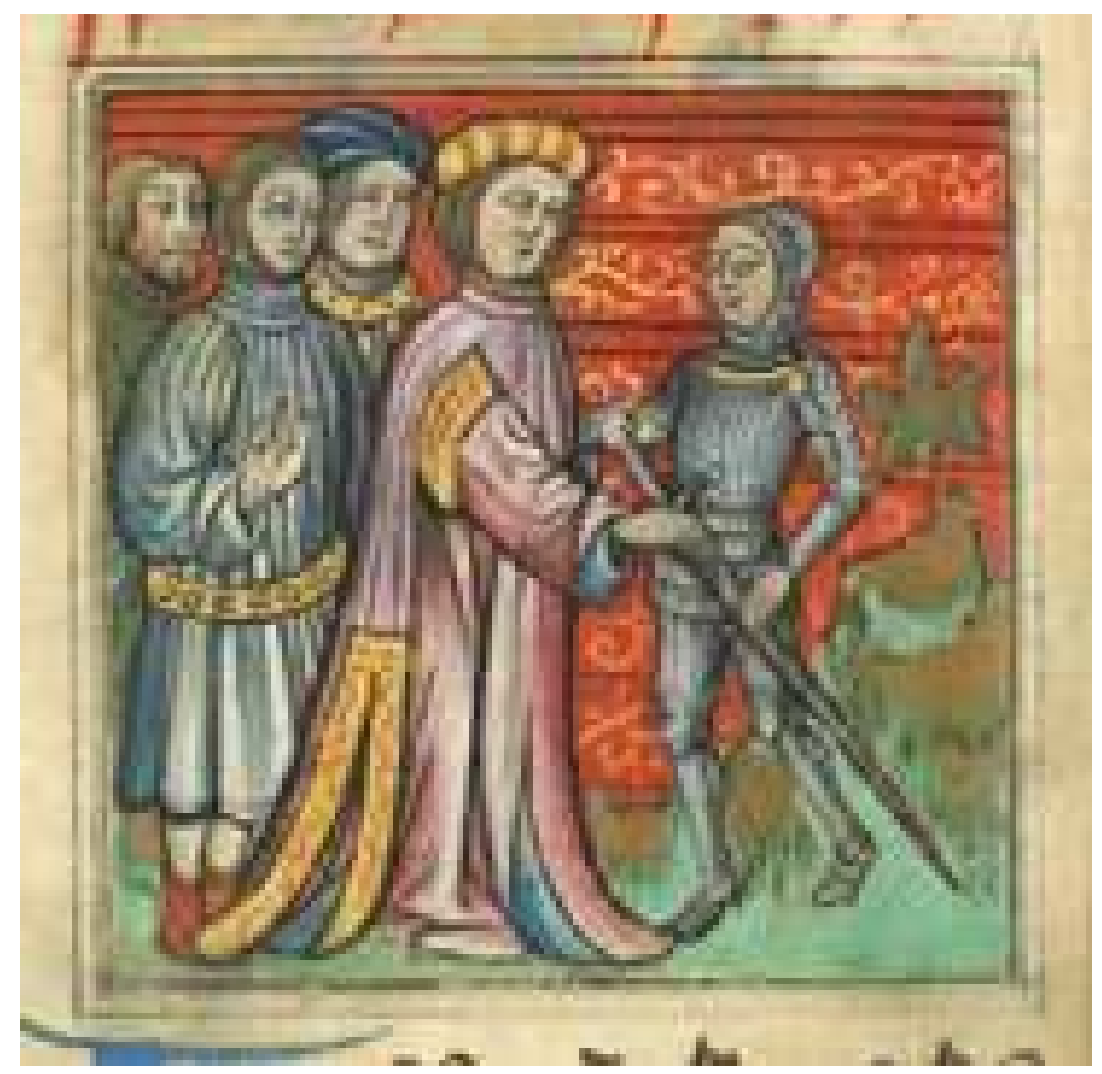

Figure 7 - Grande Chronique de Normandie, Rouen, ms Y26, fol. 6v, 1410-1420, Paris. L'adoubement de Robert le Diable.

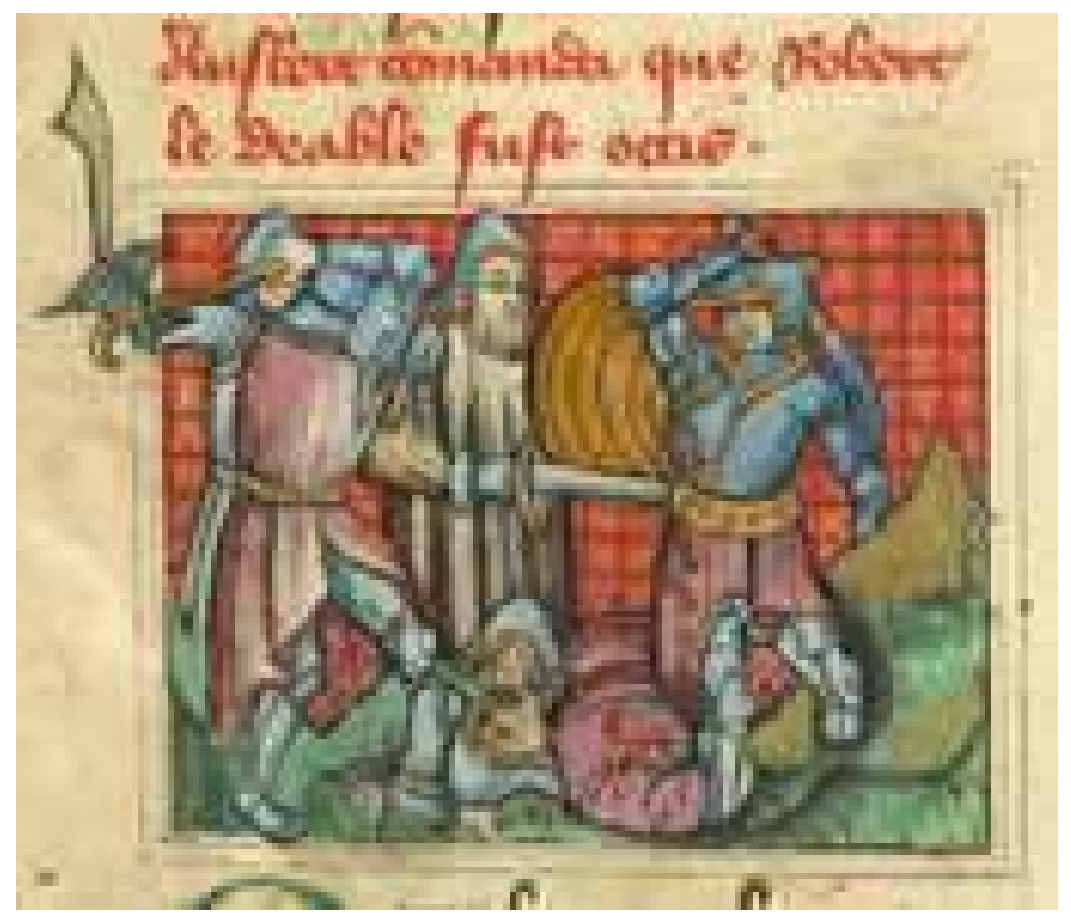

Figure 8 - Rouen, ms Y26, fol. 7v, 1410-1420, Paris. La vengeance du vicomte de Coutances. 